\author{
Каштанова Н.Ю. * Пяткова И.И.
}

ФГБУ “Институт хирургии им. А.В. Вишневского” Минздрава России, Москва, Россия

\title{
Paragangliomas of the Head and Neck: Epidemiology, Radiologic Diagnosis, Treatment
}

\author{
Kashtanova N.Yu. *, Pyatkova I.I. \\ A.V. Vishnevsky Institute of Surgery, Moscow, Russia
}

Параганглиомы головы и шеи являются крайне редкими опухолями. За весь период профессиональной деятельности врач-рентгенолог может встретить единичные случаи параганглиом, поэтому важно знать основные диагностические признаки при подозрении на данное заболевание, чтобы своевременно и с минимальным хирургическим риском выполнить оперативное вмешательство. Целью данного обзора является изучение наиболее распространенных локализаций параганглиом области головы и шеи и описание ключевых визуализационных характеристик, необходимых для правильной постановки диагноза и дифференциальной диагностики.

Ключевые слова: параганглиома, хемодектома, опухоли головы и шеи, УЗИ, КТ, МРТ.

Ссылка для цитирования: Каштанова Н.Ю., Пяткова И.И. Параганглиомы головы и шеи: эпидемиология, лучевая диагностика, лечение. Медицинская визуализация. 2017; 21 (2): 16-27. DOI: 10.24835/1607-0763-2017-2-16-27.

$$
\star \star \star
$$

Paragangliomas of the head and neck are extremely rare tumors. During the period of professional activity, a radiologist can meet single patients with parangliomas. So it is important to know the main diagnostic signs when meeting with such patients, вecause on-time diagnosis and treatment contribute to reduce surgical risk. The purpose of this review is to study the most common localizations of paragangliomas of head and neck and describe main visualization characteristics which are necessary for correct diagnosis and differential diagnosis.

Key words: paraganglioma, chemodectoma, head neck tumors, US, CT, MRI.

Recommended citation: Kashtanova N.Yu., Pyatkova I.I. Paragangliomas of the Head and Neck: Epidemiology, Radiologic Diagnosis, Treatment. Medical visualization. 2017 ; 21 (2): 16-27.

DOI: $10.24835 / 1607-0763-2017-2-16-27$.

\section{Введение}

Параганглиома (ПГ) - преимущественно доброкачественная медленнорастущая опухоль, берущая начало из клеток нервного гребня $[1,2]$. Параганглионарная система представлена структурами, рассеянными в различных органах и тканях человека в виде клубочков и играющими важную роль в гомеостазе [3, 4]. По локализации

Для корреспонденция: Каштанова Наталия Юрьевна - 117997 Москва, ул. Большая Серпуховская, д. 27, Институт хирургии им. А.В. Вишневского. Тел.: +7-985-381-46-70. E-mail: nat.y.kashtanova@mail.ru

Каштанова Наталия Юрьевна - клинический ординатор отделения рентгенологии и магнитно-резонансных исследований ФГБУ "Институт хирургии им. А.В. Вишневского" МЗ РФ, Москва; Пяткова Ирина Игоревна - младший научный сотрудник отделения ультразвуковых методов диагностики и миниинвазивного лечения ФГБУ “Институт хирургии им. А.В. Вишневского” МЗ РФ, Москва.

Contact*: Nataliya Yu. Kashtanova - 117997 Moscow, Bolshaya Serpuhovskaya str., 27, A.V. Vishnevsky Institute of Surgery. Phone: +7-985-381-46-70. E-mail: nat.y.kashtanova@mail.ru

Natalia Yu. Kashtanova - resident of radiology department of A.V. Vishnevsky Institute of Surgery, Moscow; Irina I. Pyatkova - the junior researcher of ultrasonic methods of diagnostics and miniinvasive treatment of A.V. Vishnevsky Institute of Surgery, Moscow. 
выделяют 2 большие группы ПГ. Наиболее многочисленная - в области головы и шеи, где густо располагаются нейроэндокринные скопления. Данные структуры расположены преимущественно в адвентициальной ткани артерий и вен, как правило, тесно связаны с парасимпатической нервной системой и выполняют роль хеморецепторов $[3,5,6]$. Большинство опухолей являются нефункционирующими в плане секреции катехоламинов [7]. ПГ, расположенные ниже, чаще всего исходят из мозгового вещества надпочечников (феохромоцитомы), как правило, связаны с симпатической нервной системой и способны продуцировать катехоламины $[3,8]$.

Первое упоминание о сонном клубочке сделано Von Haller в 1743 г. [9], а в 1962 г. Heller вводит понятие "гломусная опухоль". Термин "параганглиома" введен в 1903 г. Kohn, а благодаря работам G.G. Glenner и P.M. Grimley (1974) данный термин сейчас является наиболее предпочтительным для описания опухолей сонного гломуса в связи с анатомическими и физиологическими характеристиками опухоли [10].

F.G. Zak и W. Lawson описали около 20 параганглиев головы и шеи, имеющих предрасположенность к возникновению в них ПГ. Наиболее распространенными являются область сонного клубочка (каротидная хемодектома - КХД), луковица яремной вены (яремная параганглиома - ЯПГ), барабанное сплетение в области мыса (барабанная параганглиома - БПГ), ганглии блуждающего нерва (вагальная параганглиома - ВПГ) [11].

ПГ, как правило, богато васкуляризированы, что связывают с экспрессией опухолевыми клетками факторов роста эндотелия (сосудистого (VEGF) и тромбоцитарного (PD-ECGF)) [12].

Эпидемиология. На сегодняшний день ПГ остаются достаточно редкими опухолями, составляя среди всех злокачественных новообразований головы и шеи 0,016\% [13]. Наиболее частым вариантом является КХД [14-16], поэтому основные эпидемиологические данные основаны на изучении именно КХД.

ПГ диагностируются в возрасте 40-50 лет $[13,17]$, отмечается некоторое преобладание женщин [7, 17-19].

M.S. Sajid и соавт. выделяют 3 формы КХД: семейная, спорадическая, гиперпластическая. Последняя характерная для пациентов с хронической обструктивной болезнью легких, врожденными цианотическими пороками сердца и для жителей горной местности, что связывают с хронической гипоксией [7]. Семейная форма ассоциирована с мутацией генов, кодирующих фермент сукцинатдегидрогеназу (SDHB, SDHC, SDHD,
SDHAF2) [20, 21], митохондриальный фермент, отвечающий за превращение сукцината в фумарат в цикле Кребса. Инактивация фермента приводит к кумуляции сукцината и патологической активации пути гипоксии-ангиогенеза, что объясняет гиперваскуляризацию. Также семейная форма ассоциирована с рядом наследственных заболеваний (МЭН II типа, фон Гиппеля-Линдау, нейрофиброматоз 1-го типа) [16] и встречается в 30-50\% случаев [1]. Таким образом, пациенты с диагностированными ПГ в возрасте менее 50 лет, с семейными случаями ПГ, а также с множественным поражением должны пройти генетическое тестирование [17, 22]. Спорадическая форма характеризуется наличием ПГ любой локализации при отсутствии семейного анамнеза и факторов, характерных для гиперпластической формы.

Множественные ПГ могут быть синхронными (диагностироваться одновременно) и метахронными [15]. По данным различных исследований, доля первично множественных каротидных ПГ составляет в среднем 10-25\% [16, 19, 23, 24]. Билатеральная локализация отмечается в среднем в 4,4\% случаев спорадического поражения и 31,8\% семейного [25]. Множественные ПГ могут происходить как из одного субстрата (например, каротидные), так и в сочетании с другими (вагальной или яремного гломуса) [21, 19]. К примеру, в исследовании A. Szymańska и соавт. (2015) приведено клиническое наблюдение пациента с множественным двусторонним поражением: наличие каротидной, вагальной, яремной ПГ с одной стороны, каротидной с другой стороны и метахронное поражение аортопульмональной локализации) [15].

ПГ - опухоли преимущественно доброкачественные и характеризуются медленным ростом около 0,2 см/год $[18,26]$. Однако встречается и злокачественное течение ПГ, частота таких форм может достигать 4\% [16]. По радиологическим или гистологическим данным предсказать риск неблагоприятного течения затруднительно. Достоверными признаками злокачественности ПГ считают обнаружение инвазивного роста и метастазов (как правило, это лимфатические узлы шеи, реже - легкие, печень, кости) $[7,12,13]$. Регионарные и отдаленные метастазы могут диагностироваться одновременно с первичной опухолью или выявляться в различные сроки после резекции $[4,13,19]$. Данные исследования K. Papaspyrou и соавт. (2012) указывают на высокое сродство злокачественных форм и наличие генетических нарушений у пациентов [19].

Рассмотрим основные варианты ПГ головы и шеи. 


\section{Каротидная параганглиома (хемодектома)}

Как было сказано выше, ПГ сонного гломуса является самой распространенной формой ПГ головы и шеи (более 50\%). Опухоль развивается из сонного клубочка, расположенного в адвентиции в области бифуркации общей сонной артерии (ОСА), при больших размерах может интимно контактировать с боковой стенкой глотки, трахеей, блуждающим, языкоглоточным нервами, щитовидной железой, внутренней яремной веной [1416].

По степени контакта опухоли с каротидными сосудами Л.А. Атанасян (1969) выделяет 4 типа КХД [27]:

1 - опухоль раздвигает сонные артерии и довольно рыхло связана с адвентицией;

2 - опухоль муфтообразно охватывает наружную сонную артерию (НСА);

3 - опухоль муфтообразно охватывает внутреннюю сонную артерию (ВСА);

4 - опухоль охватывает и сдавливает бифуркацию и все ветви сонной артерии.

Основной жалобой пациента с КХД является наличие объемного пульсирующего безболезненного образования в области угла нижней челюсти $[7,18,28]$.

Лучевая диагностика. Основной целью инструментальных методов исследования являются подтверждение диагноза, оценка распространенности опухоли, выявление причастности окружающих нервных образований и определение степени злокачественности процесса.

При любом методе исследования КХД представляют собой гиперваскулярное объемное образование, расположенное в проекции бифуркации ОСА и смещающее ВСА заднелатерально и НСА переднемедиально и распространяющееся краниально от области бифуркации ОСА [29].

При ультразвуковом исследовании (УЗИ) опухоль имеет четкие контуры, гипо- или изоэхогенную структуру по отношению к эхогенности окружающих мышц, гиперэхогенную капсулу толщиной до 3 мм, при дуплексном сканировании определяется выраженный внутриопухолевый кровоток (рис. 1, 2) [7, 30, 31]. При малых размерах опухоль может не иметь гемодинамически значимого влияния на просвет сосуда, но при значительных размерах может значимо деформировать ход сосуда или сужать его просвет (при муфтообразном росте опухоли), что приводит к характерным проявлениям ультразвуковой картины и допплерографическим изменениям (рис. 3) [32]. При дуплексном сканировании определяется асимметрия скорости кровотока с ее увеличением на стороне поражения по сравнению с противоположной стороной, она может повышаться более чем на $60 \%$ [33].

Компьютерная томография (КТ) позволяет определить размеры, плотность и синтопию образования, а также судить о степени вовлечения в процесс сонных артерий и костных структур [33]. Основное значение в дифференциальной диагностике КХД от других опухолей в области бифуркации каротидных артерий имеет артериальная фаза, а именно раннее и выраженное (от 104 до 250 ед.Н, $174 \pm 51$ ед.Н) накопление хемодектомой контрастного вещества (рис. 4) [29]. Также артериальная фаза позволяет четко определить нижний полюс опухоли (обычно на уровне бифуркации ОСА или на 0,5-1,5 см ниже) и расстояние от опухоли до основания черепа, а также взаимоотношение опухоли с бифуркацией и ВСА [29]. Расширение бифуркации ОСА - типичный признак каротидной ПГ (рис. 5). В крупных опухолях контрастирование может быть гетерогенным вследствие участков гиалиноза, фокальных тромбозов или кровоизлияний [29, 34]. На основании данных KT возможно построение 3D-реконструкций, что позволяет наглядно показать взаимоотношение опухоли с сонными артериями [35].

Ангиографию используют для оценки расположения опухоли по отношению к ВСА и внутренней яремной вене, определения их проходимости, а также визуализации основной кровоснабжающей опухоль артерии, которой в большинстве случаев является восходящая глоточная артерия (ветвь НСА) [36], и коллатералей [14]. КХД при контрастировании представляет собой конгломерат переплетающихся между собой сосудов [33] и имеет характерный вид "лиры”, отодвигая НСА и ВСА друг от друга (рис. 6), в то время как ВПГ, как правило, расположена выше и смещает как наружные, так и внутренние сонные артерии переднемедиально [14]. Зачастую ангиография применяется не в качестве диагностического метода, а как начальный этап процедуры эмболизации.

Классический симптом "соли и перца" хемодектомы при MPT впервые описан W.L.G. Olsen и соавт. в 1987 г. [37]. Он обусловлен потоковыми дефектами в крупных внутриопухолевых сосудах: гипоинтенсивный компонент в виде "перца" чередуется с гиперинтенсивными участками в виде "соли" (из-за медленного кровотока или кровоизлияний) и отмечается при всех магнитно-резонансных последовательностях. Опухоль на Т1взвешенном изображении (ВИ) имеет преимущественно изоинтенсивный сигнал по отношению к мышцам, тогда как на Т2ВИ - преимущественно гиперинтенсивный. На постконтрастных изображениях отмечается выраженное накопление конт- 

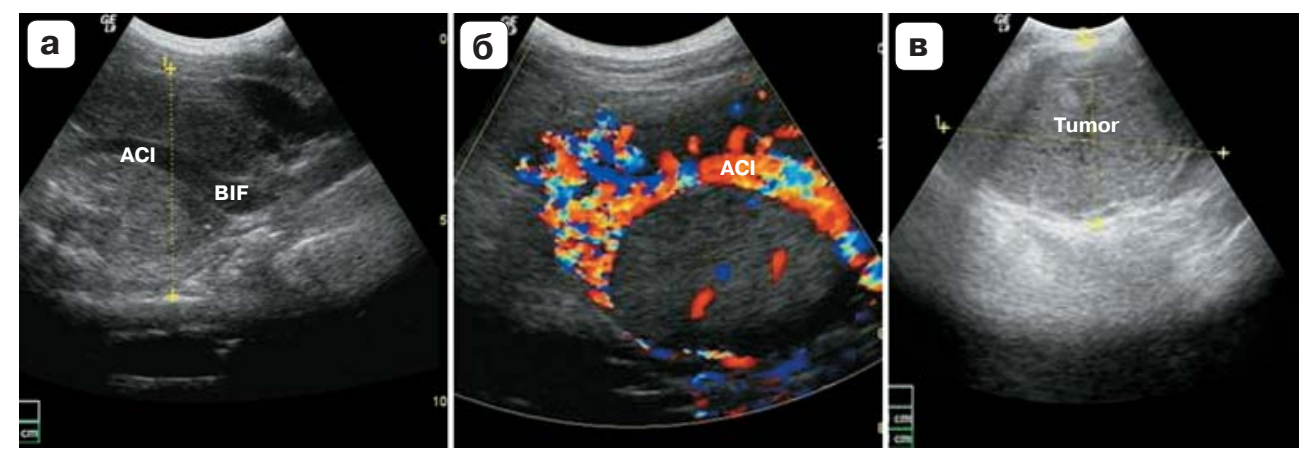

Рис. 1. Пациентка 50 лет. УЗ-изображения: в области бифуркации правой ОСА, раздвигая артерии, определяется объемное солидное, неоднородное по структуре образование (а), гиперваскулярное (б), размерами $12 \times 7$ см (в). Бифуркация OCA, HCA и BCA находятся в структуре опухоли. ACI - внутренняя сонная артерия, BIF - бифуркация OCA, Tumor - опухоль.
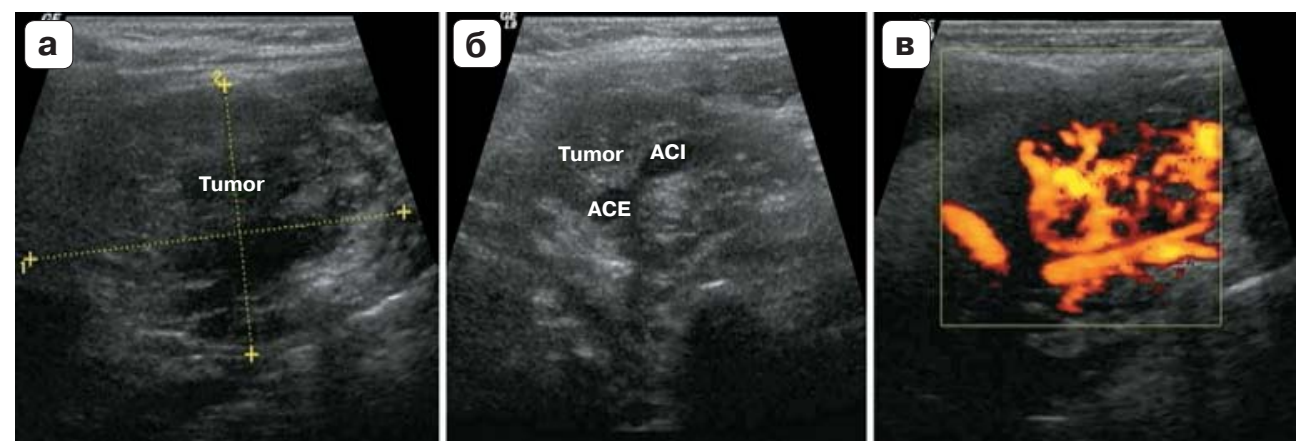

Рис. 2. Пациентка 48 лет. УЗ-изображения: в области бифуркации ОСА определяется солидное образование с четкими ровными контурами, неоднородное по структуре, размерами 5,2 × 3,4 см (а). Дистальный отдел ОсА с бифуркацией, ВСА и НСА располагаются внутри образования (б). Образование гиперваскулярно (в), в структуре определяются преимущественно артерии. ACI - внутренняя сонная артерия, ACE - наружная сонная артерия, Tumor - опухоль.
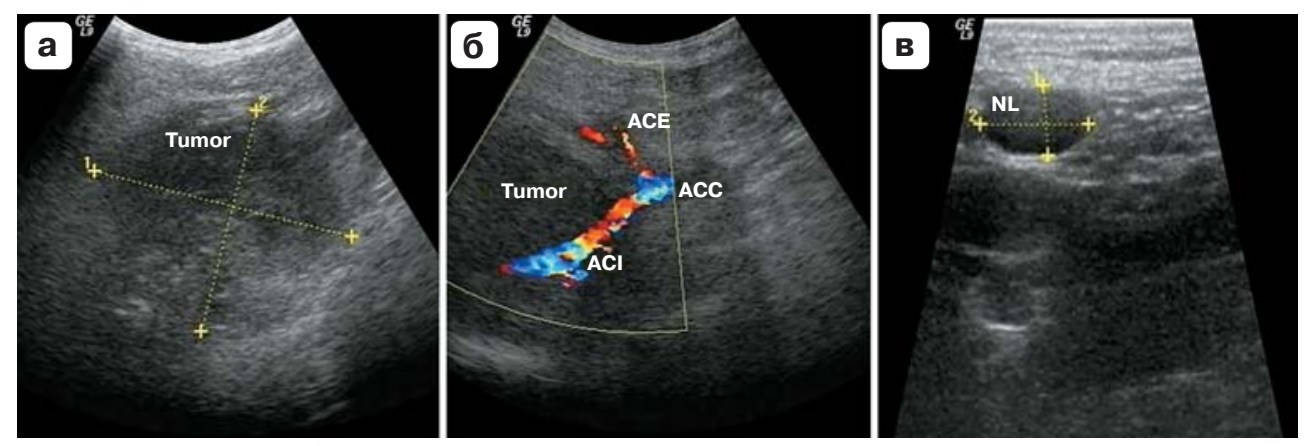

Рис. 3. Пациент 45 лет. УЗ-изображения: визуализируется объемное образование области бифуркации ОсА, имеющее неоднородную солидную структуру, четкие ровные контуры, размерами $6 \times 5$ см (а). Взаимоотношение с магистральными сосудами: ВСА оттеснена кзади, проходит по заднему контуру образования, имеет гемодинамически незначимый плавный изгиб в области образования и тотчас за ним. НСА располагается кпереди от образования (б). Определяются множественные лимфатические узлы, расположенные по ходу кивательной мышцы, больший из которых размерами 2,4 см (в). 

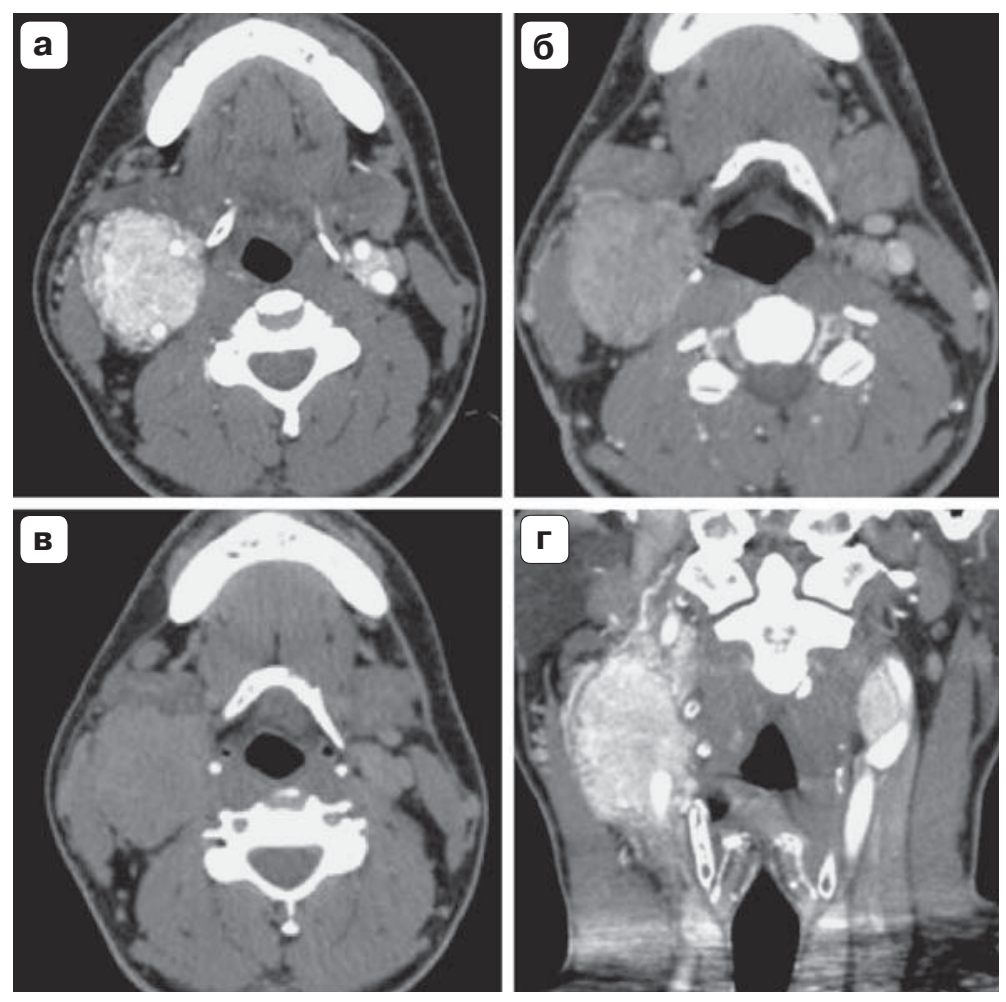

Рис. 4. Пациент 45 лет. МСКТ-изображения с внутривенным контрастным усилением. Аксиальная (а-в) и фронтальная (г) реконструкции. Справа в области каротидной бифуркации определяется образование размерами 3,7 × 4,8 × 7,0 cм, неправильной бугристой формы, мягкотканной плотности, выраженно накапливающее контрастное вещество в артериальную фазу (а) до 212 ед.Н, в венозную фазу (б) до 169 ед.Н, в отсроченную фазу (в) до 78 ед.Н. С латеральной стороны образование несколько деформирует поверхность шеи, впереди несколько смещает вперед подчелюстную слюнную железу и медиальную крыловидную мышцу. Бифуркация правой ОСА внутри опухоли. ВСА на протяжении 35 мм проходит в структуре опухоли и направляется к задней стенке. По задней стенке опухоль охватывает ВСА на $90 \%$ ее окружности (г). НСА на протяжении 3,2 см от развилки проходит внутри опухоли, после чего выходит на переднюю поверхность. В бифуркации левой ОСА определяется подобное образование меньших размеров 1,3 × 1,6 × 1,7 cм, охватывающее внутреннюю и наружную сонные артерии не более чем на 1/3 окружности (г) (двусторонняя каротидная параганглиома).
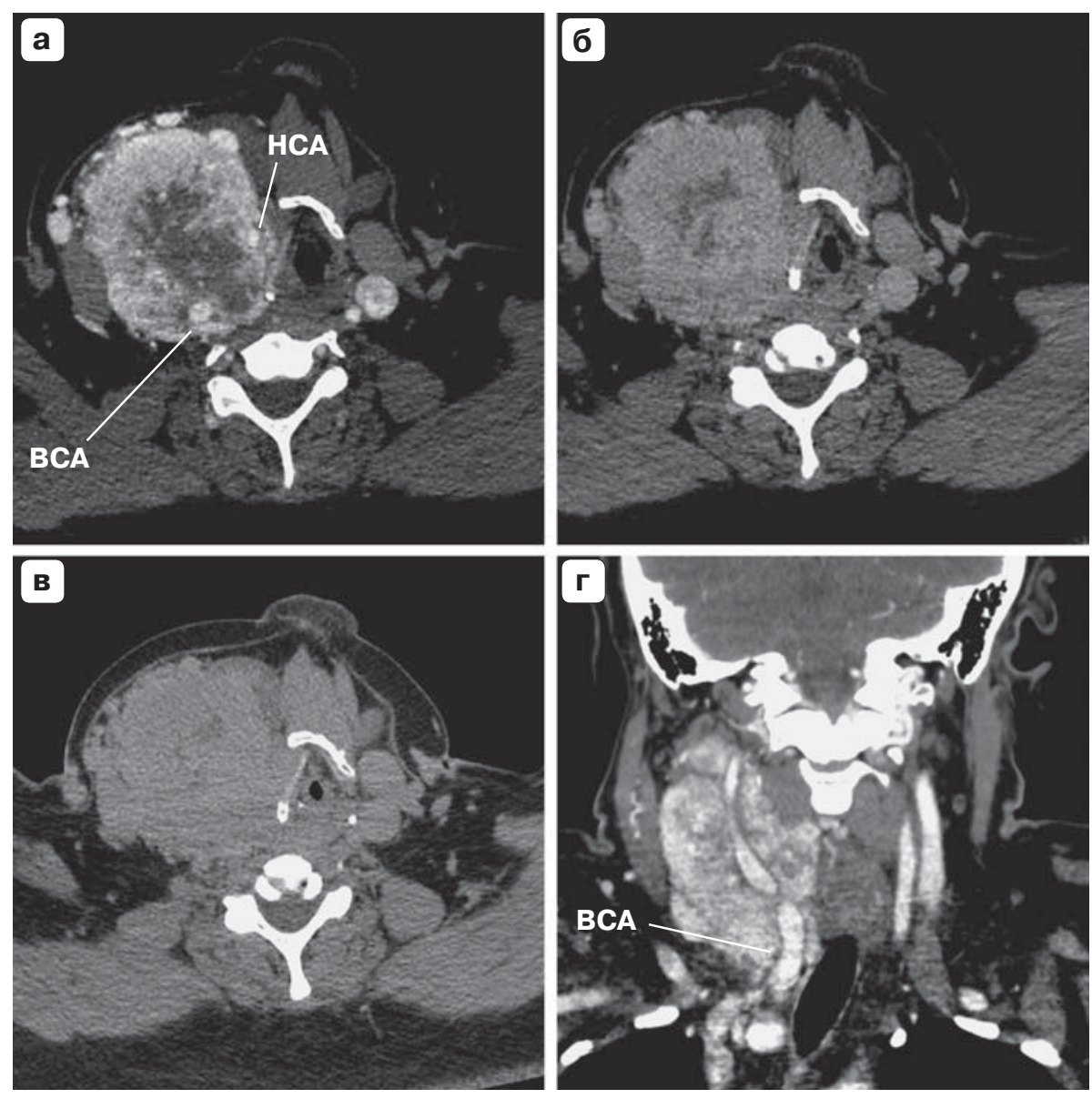

Рис. 5. Пациентка 50 лет. МСКТ-изображения с внутривенным контрастным усилением. Аксиальная (а-в) и фронтальная (г) реконструкции. Начиная от уровня правой доли щитовидной железы и до основания черепа (уровень $\mathrm{C}_{\text {I }}$ ) определяется гиперваскулярное образование размерами 7,8 ×6,8 × 10,3 cм, неоднородной структуры за счет наличия участка низкой плотности в центре. В артериальную фазу (а) образование интенсивно накапливает контрастный препарат до 180 ед.Н, в венозную фазу (б) до 97 ед.Н, в отсроченную до 79 ед.Н. Образование сдавливает правую долю щитовидной железы, смещает трахею и ротоглотку влево. Признаков прорастания в них не определяется. Опухоль по всей окружности охватывает бифуркацию ОСА, ВСА и НСA $(\mathrm{a}, \mathrm{r})$. 


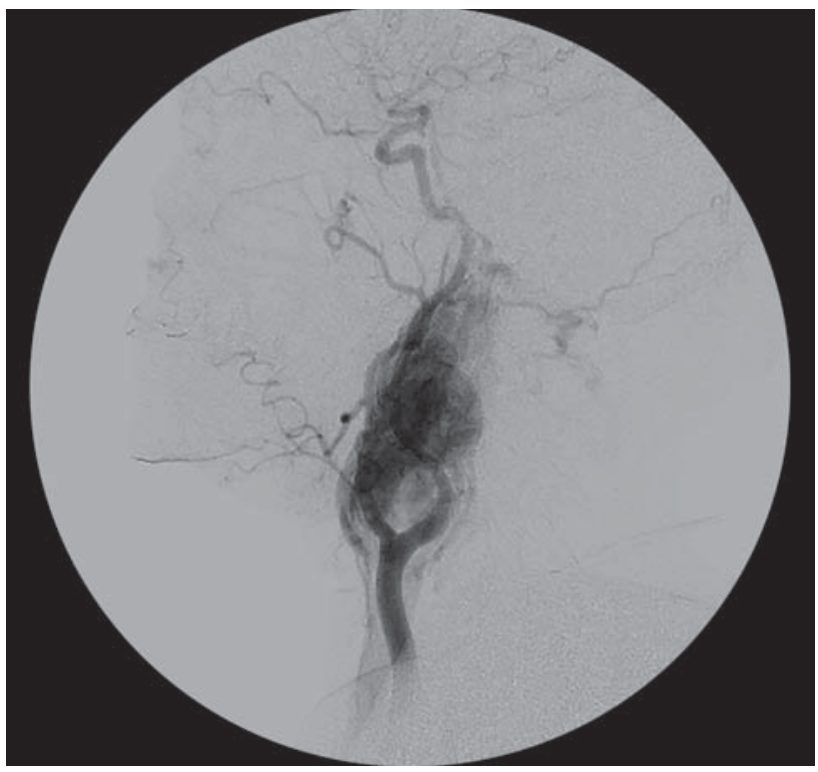

Рис. 6. Пациент 57 лет. Селективная ангиография брахеоцефального ствола, общей, наружной и внутренней сонных артерий. БЦА, ОСА и ВСА с ровными контурами, контрастируются гомогенно. НСА и ее ветви сильно извиты и деформированы. В области левой каротидной бифуркации контрастируется гиперваскулярное образование с артериовенозным сбросом на 2-5-й секунде. Образование получает кровоснабжение из бассейна НСА и ветвей щитошейного ствола.

растного препарата [38]. G. Gravel и соавт. считают, что использование режима 3D angio-MR в артериальную фазу контрастирования достаточно для обнаружения ПГ любой локализации, а добавление постконтрастных Т1ВИ дает дополнительную топическую информацию, что полезно для планирования оперативного лечения или радиотерапии [39]. Сокращенный режим MP-сканирования (постконтрастные 3D angio-MR изображения и fast spin-echo T1BИ) также полезен для скрининга бессимптомных пациентов-носителей мутаций группы генов SDHx [40].

\section{Вагальная параганглиома}

ВПГ является второй по частоте параганглиомой головы и шеи (10-15\%) и представляет собой доброкачественную сосудистую опухоль из нодозного или яремного ганглиев блуждающего нерва [16]. В 1/3 случаев диагностируется множественное поражение, у 80\% пациентов из данной группы отмечается наследственная предрасположенность [41], двустороннее поражение наблюдается в 7\% случаев [15].

ВПГ локализуются в передне- или заднебоковом окологлоточном пространстве, верхний полюс опухоли доходит до основания черепа, а нижний может достигать бифуркации ОСА, что объясняет их интимное прилегание к блуждающим, подъязычным и языкоглоточным нервам, ВСА [16]. Дифференциально-диагностическим признаком ВПГ в отличие от КХД является переднемедиальное смещение ВСА с оттеснением кзади и латерально внутренней яремной вены без вовлечения каротидной бифуркации [42].

Как правило, ВПГ безболезненны и при осмотре представлены объемным пульсирующим образованием, расположенным в заднелатеральном отделе глотки. Возможны сопутствующие симптомы, возникающие вследствие сдавления опухолью нервов: паралич голосовых связок с дисфонией, синдром Горнера [16].

Лучевая диагностика. ВПГ представлены образованием овоидной формы, расположенным в области сонных артерий, причем ВСА смещается опухолью переднемедиально (рис. 7) (отличительный признак при сравнении с КХД), а внутренняя яремная вена - заднелатерально без вовлечения каротидной бифуркации [42].

При УЗИ ВПГ определяются как гиперваскулярные объемные образования овоидной формы, расположенные в области сонных артерий, с определенной синтопией, описанной выше [43]. Структура и эхогенность не отличаются от таковых, описанных для КХД $[43,44]$.

КT - наиболее информативный метод при малых размерах опухоли. Характеристики аналогичны КХД [42]. При больших размерах опухоли, достигающей основания черепа, отсутствие деструкции и расширения яремного отверстия служит дифференциально-диагностическим признаком в отличие от ЯПГ [15].

МРТ-картина аналогична КХД $[42,45]$.

\section{Яремная параганглиома}

Опухоль локализуется в области яремного отверстия затылочной кости и исходит из барабанной ветви языкоглоточного нерва (нерв Якобсона) или ушной ветви блуждающего нерва (нерв Арнольда), распространяется в сторону сосцевидных ячеек, сосудистых и нервных каналов $[46,47]$, евстахиевой трубы [48]. При проникновении в полость среднего уха ПГ получает название яремнобарабанная (рис. 8) [49].

Пациентов с ЯПГ беспокоят пульсирующий шум в ушах и наличие ретротимпанального опухолевидного образования. Может приводить к нейропатии языкоглоточного, блуждающего, добавочного нервов и вызывать кондуктивную тугоухость, боль в ухе, головокружение, охриплость [16].

Лучевая диагностика. КТ на ранних стадиях выявляет объемное образование, деформирующее 

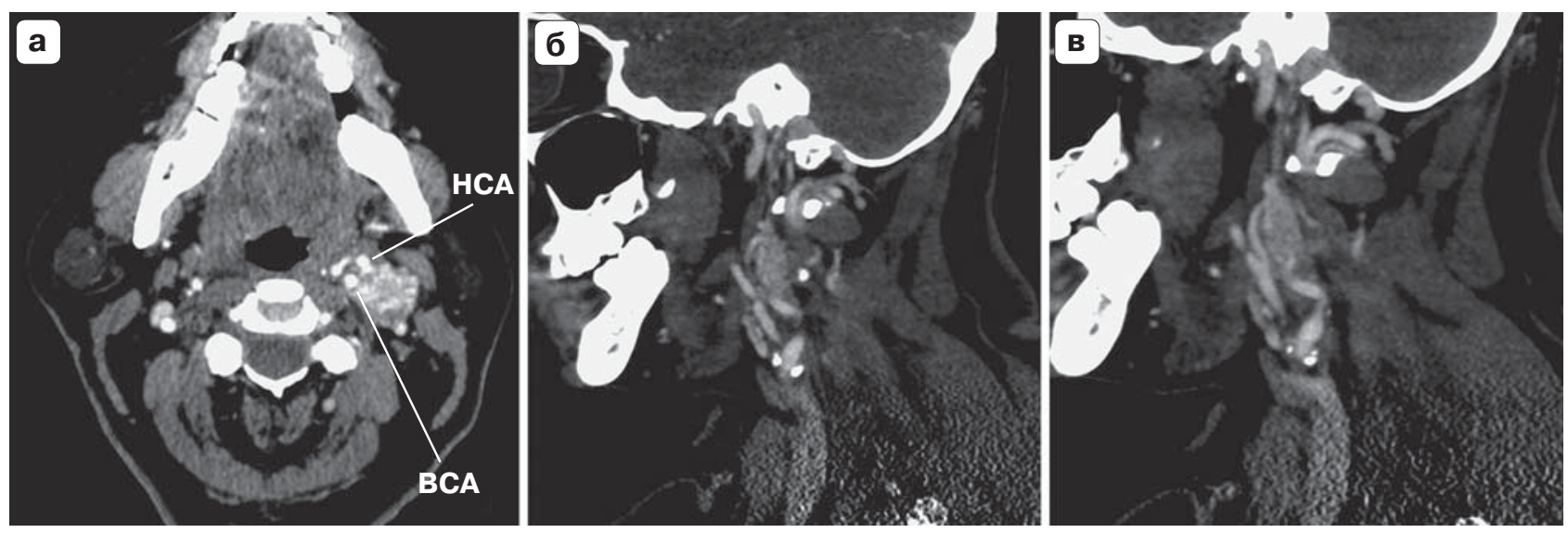

Рис. 7. Пациентка 53 лет. МСКТ-изображения с внутривенным контрастным усилением. Аксиальная (а) и сагиттальная (б, в) реконструкции. Латеральнее ВСА визуализируется образование размерами 2,6 × 1,9 × 2,9 см, неоднородное, неравномерно накапливающее контрастный препарат. Левая ВСА граничит с образованием на половину окружности, смещена кпереди и медиально. НСА на протяжении 3 мм контрактирует с образованием на половину окружности, отдает коллатерали к образованию.
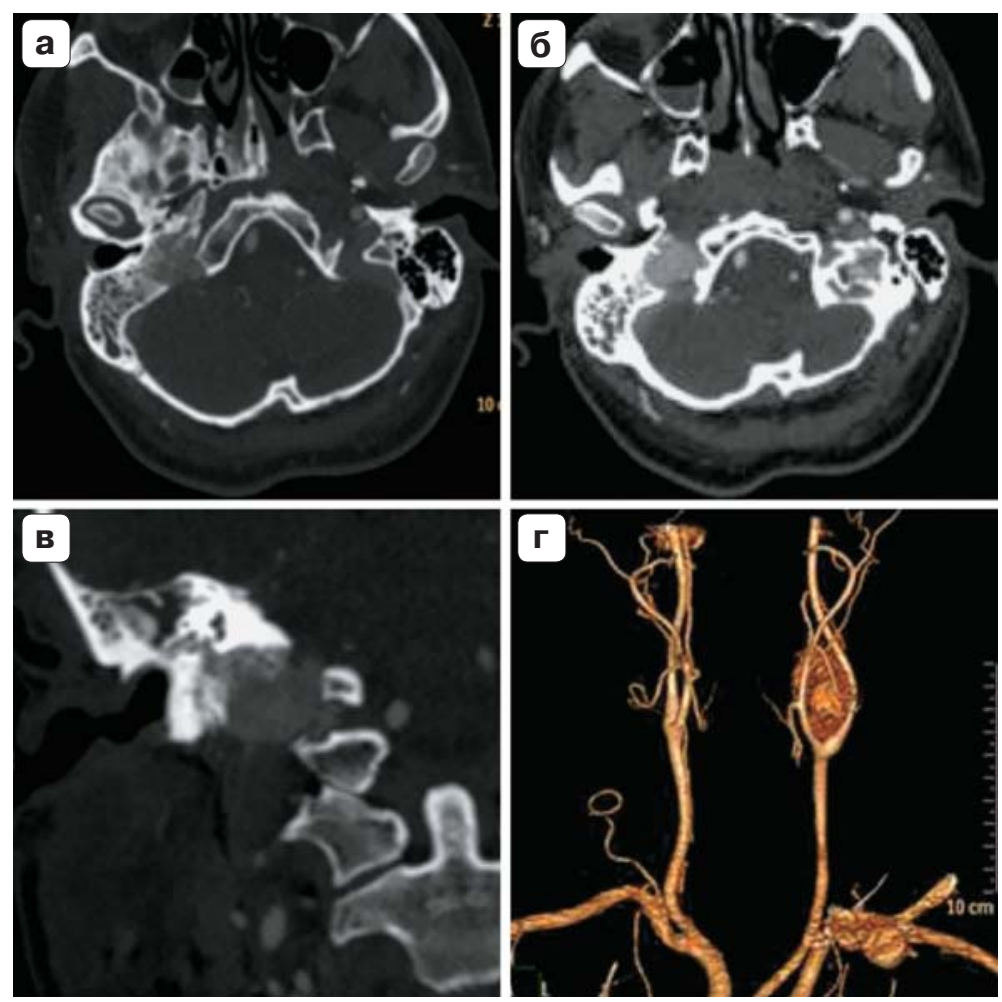

Рис. 8. Пациент 56 лет. МСКТ-изображения с внутривенным контрастным усилением. Аксиальная (а, б), сагиттальная (в) и 3D-реконструкции (г). К нижней стенке барабанной полости справа прилежит образование овальной формы с дольчатыми контурами размерами до 1,9 × 1,3 × 1,7 см, выраженно накапливающее контрастное вещество в артериальную фазу до 290 ед.Н, разрушающее прилежащие отделы височной кости и проникающее в полость среднего уха. Слева в области каротидной бифуркации, раздвигая ее, визуализируется аналогичное образование неправильной овальной формы размерами 3,6 × 3,0 × 4,0 см. Множественное поражение: каротидная параганглиома слева и яремно-барабанная параганглиома справа. 

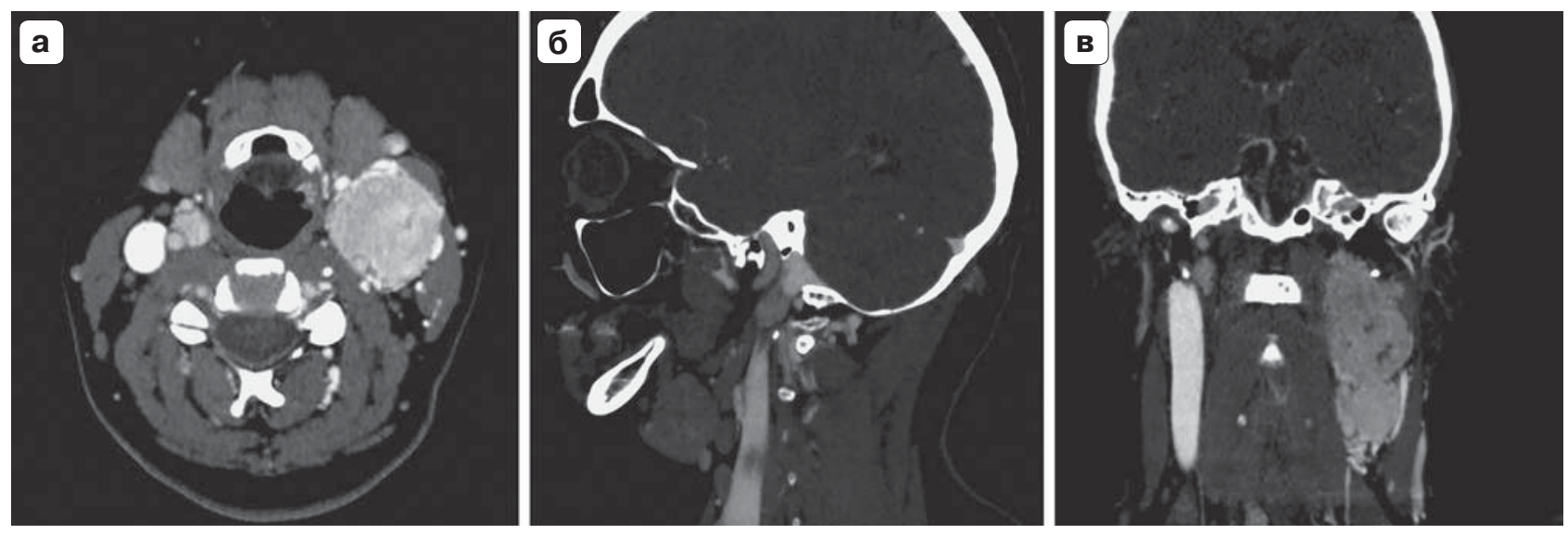

Рис. 9. Пациентка 37 лет. МСКТ-изображения с внутривенным контрастным усилением. Аксиальная (а), сагиттальная (б) и фронтальная (в) реконструкции. Слева в области бифуркации ОСА определяется образование с ровными четкими, несколько бугристыми контурами (а, в). В артериальную фазу образование интенсивно накапливает контрастный препарат до 224 ед.Н с сохранением гиподенсных участков звездчатой формы в центральной части (каротидная хемодектома). Аналогичные образования определяются в бифуркации правой ОСА размерами $1,1 \times 1,1 \times 1,2$ см и по внутреннему контуру внутренней яремной вены на уровне атлантоокципитального сочленения размерами 0,8 $\times$ $\times 1,2 \times 1,5$ см (б) (яремная параганглиома). Параметры контрастирования образований аналогичные с образованием на контралатеральной стороне.

яремное отверстие. Прогрессирующий рост опухоли приводит к деструкции по типу "изъеденностью молью” костных структур яремного отверстия и сосцевидного отростка [15]. Разрушение цепи слуховых косточек также является характерным симптомом (рис. 9) [50].

При МРТ опухоли размерами более 2 см дают симптом "соли и перца". На Т2ВИ опухоли обладают гетерогенным сигналом от изо- к гиперинтенсивному, на Т1ВИ с контрастированием - выраженное накопление контрастного препарата [15].

\section{Барабанная параганглиома}

Самая распространенная опухоль среднего уха [49]. Как правило, не вовлекает яремное отверстие. Представляет собой небольшое изолированное поражение мыса улитки в барабанной полости, в области которого расположены гломусы нижнего барабанного нерва (Якобсона) - ветви языкоглоточного нерва. БПГ также могут распространяться в ячейки сосцевидного отростка, евстахиеву трубу и носоглотку. В отличие от ЯПГ, при данном типе нехарактерна деструкция слуховых косточек [41].

Основные жалобы пациентов: шум в ухе, кондуктивная тугоухость, паралич лицевого нерва [51].

Лучевая диагностика. Основное значение имеет KT, которая выявляет небольшое округлое образование с плоским основанием на мысе улитки, выступающее в мезотимпанум [41].

MPТ играет важную роль при оценке распространения опухоли интракраниально и на основание черепа. На бесконтрастных Т1ВИ опухоли да- ют интенсивность сигнала с характерным симптомом "соли и перца" [49, 52].

\section{Лечение параганглиом головы и шеи}

КХД после их выявления должны быть удалены, поскольку рост образования сопряжен не только с воздействием на окружающие структуры и расширением спектра симптомов, но и повышением риска оперативного вмешательства, так как область шеи богата крупными сосудисто-нервными структурами [36, 53].

Первые попытки удаления опухоли предпринимались еще в XIX веке, но были неудачны (1880 - Reigner, гибель пациента (цит. по Lahey F.H. и Warren K.W., 1951 г. [54]); 1886 - Maydl, инсульт в раннем послеоперационном периоде (цит. по Byme J.J., 1958 г. [55])). Первое успешное лечение хемодектомы выполнено Albert в 1889 г. [56], a G. Gordon-Taylor описал метод безопасной субадвентициальной диссекции в 1940 г. [57].

Для оценки пери- и послеоперационного риска существует классификация W.R. Shamblin и соавт. (1971), оценивающая наличие или отсутствие двух основных признаков: циркулярного окружения опухолью каротидных сосудов и инфильтрации стенки сосуда [58]. Однако она имеет ряд ограничений. Во-первых, окончательное суждение о степени инфильтрации зачастую возможно только интраоперационно, что снижает ценность классификации при предоперационном планировании. Во-вторых, большее внимание в классификации уделяется именно размерам опухоли, чем ее вза- 
Таблица 1. Модифицированная классификация каротидных параганглиом по W.R. Shamblin и соавт. [53]

\begin{tabular}{c|c|c|c}
\hline $\begin{array}{c}\text { Tип } \\
\text { по W.R. Shamblin }\end{array}$ & Размер & $\begin{array}{c}\text { Окружение или инфильтрация } \\
\text { стенки сосуда }\end{array}$ & Удаление опухоли \\
\hline I & $<4 \mathrm{~cm}$ & Нет & Благоприятное \\
II & $>4 \mathrm{~cm}$ & Частично прилежит & Затрунено \\
IIIb = I, II, III + & $>4 \mathrm{~cm}$ & Плотно прилежит & Осложнено в связи \\
Лнфильтративный рост & & & $\begin{array}{c}\text { с неободимостью выполнения } \\
\text { реконструктивнй } \\
\text { операции на сосудах }\end{array}$ \\
\hline
\end{tabular}

Таблица 2. Классификация каротидных хемодектом на основании структуры опухоли [59]

\begin{tabular}{|c|c|c|c|}
\hline Тип & Структура опухоли & Интраоперационные особенности & Резекция \\
\hline$A$ & $\begin{array}{l}\text { Мягкотканная } \\
\text { опухоль с губчатой } \\
\text { структурой }\end{array}$ & $\begin{array}{l}\text { Часто ассоциируется с тяжелым } \\
\text { интраоперационным кровотечением; } \\
\text { легко отделяется от соседних сосудов }\end{array}$ & Обычно полная \\
\hline B & Плотная (эластичная) & $\begin{array}{l}\text { Менее выряженное кровотечение; } \\
\text { плотное прилегание к соседним } \\
\text { сосудам и трудности выделения }\end{array}$ & Возможна полная резекция \\
\hline $\mathrm{C}$ & $\begin{array}{l}\text { Очень плотная } \\
\text { (гравиеподобная) }\end{array}$ & $\begin{array}{l}\text { Выраженность кровопотери меньше, } \\
\text { чем при типах А и В; очень плотный } \\
\text { контакт с окружающими структурами }\end{array}$ & $\begin{array}{l}\text { Полная резекция может быть } \\
\text { невозможна. Необходима частичная } \\
\text { резекция/удаление опухоли с } \\
\text { прилегающими сосудами }\end{array}$ \\
\hline $\mathrm{D}$ & $\begin{array}{l}\text { Солидно- } \\
\text { мягкотканная } \\
\text { (промежуточный тип } \\
\text { между В и С) }\end{array}$ & $\begin{array}{l}\text { Солидный компонент тесно прилежит } \\
\text { к сосудистым структурам }\end{array}$ & $\begin{array}{l}\text { Возможна полная или частичная } \\
\text { резекция }\end{array}$ \\
\hline
\end{tabular}

имоотношениям с сосудами. И хотя при большем размере (III тип по W.R. Shamblin) предполагается большая частота инфильтрации сосудов, эта зависимость не всегда прямая [53].

K. Luna-Ortiz и соавт. (2006) предложили дополнить классификацию по W.R. Shamblin, в которой сохранилось разделение на типы в зависимости от размера опухоли и степени контакта с сосудами. При этом IIla тип в новой классификации равноценен III типу по W.R. Shamblin, а в IIIb тип включены опухоли любого размера, но при наличии инфильтративного роста в стенку сосуда (при клиническом или гистологическом подтверждении) (табл. 1) [53].

Ү. Ма и соавт. (2014) также считают, что исход операции при опухолях в области сонных артерий зависит не только от размера опухоли, но и от ее структуры, и предложили свою классификацию (табл. 2). Согласно ей, мягкотканные опухоли (тип А) зачастую могут быть полностью иссечены с сохранением ВСА, В то время как солидные (типы B, C и D) сопряжены с большей сложностью операции [59].

В некоторых ситуациях перед операцией предпринимается попытка эндоваскулярной эмболизации $[36,60]$. Мнения на этот счет различны.
По данным одних исследователей, рутинная предоперационная эмболизация полезна для облегчения удаления опухоли, так как снижает ток крови в опухоли и уменьшает размеры образования $[61,62]$. С другой стороны, ряд исследований показал отсутствие достоверных различий в объеме кровопотери, длительности операции и риске неврологических осложнений при сравнении групп пациентов с предоперационной эмболизацией и без нее [36, 63, 64].

Лучевая терапия в настоящее время используется в основном у пациентов, которым противопоказано хирургическое лечение по причине выраженного инфильтративного роста, при множественном поражении, высоком оперативном риске, а также при обнаружении остаточной опухоли в месте резекции и при рецидивах $[14,65,66]$. По данным метаанализа Z.D. Guss и соавт. (2011), радиотерапию следует применять в качестве первичного метода лечения ЯПГ вследствие более агрессивного течения и высокой частоты рецидива после резекции [67].

После резекции ПГ шеи рекомендовано регулярное диспансерное наблюдение больных, что позволит своевременно выявить рецидивы и метастазы опухоли. 


\section{Заключение}

ПГ головы и шеи мало распространены в популяции. Опухоли каротидного гломуса являются самой частой формой ПГ данной локализации, на втором месте стоят вагальные и яремные ПГ. При множественных ПГ отмечается большая частота семейных форм. Опухоль имеет длительный бессимптомный период, однако при значительном росте может вызывать сдавление окружающих сосудисто-нервных структур, гортани, костей, других органов шеи, что снижает качество жизни пациента. Основным диагностическим признаком является обнаружение объемного образования в области скопления параганглиев, высоковаскуляризированного, что проявляется изменением показателей при дуплексном сканировании, выраженным накоплением контрастного препарата в артериальную фазу при КТ или МРT, а также классическим МР-симптомом в виде "соли и перца". ПГ считается злокачественной при обнаружении локальных (в лимфатические узлы) и отдаленных метастазов. Знание основных характеристик ПГ позволит своевременно и правильно поставить диагноз и запланировать эффективное лечение.

\section{Список литературы / References}

1. Meli G.A., Chiaramonte R., Cavallaro T., Puglisi C., Pero G. Carotid body paraganglioma. Diagnosis and treatment by angiography. Neuroradiol. J. 2006; 19: 645-648.

2. Chapman D.B., Lippert D., Geer C.P., Edwards H.D., Russell G.B., Rees C.J., Browne J.D. Clinical, histopathologic, and radiographic indicators of malignancy in head and neck paragangliomas. Otolaryngol. Head Neck Surg. 2010; 143: 531-537.

DOI:10.1016/j.otohns.2010.05.031.

3. Boedeker C.C., Ridder G.J., Schipper J. Paragangliomas of the head and neck: diagnosis and treatment. Familial Cancer. 2005; 4: 55-59.

DOI:10.1007/s10689-004-2154-z.

4. Mediouni A., Ammari S., Wassef M., GimenezRoqueplo A.P., Laredo J.D., Duet M., Tran Ba Huy P., Oker N. Malignant head/neck paragangliomas. Comparative study. Eur. Ann. Otorhinolaryngol. Head Neck Dis. 2014;131(3):159-166. DOI:10.1016/j.anorl.2013.05.003.

5. Kaklikkaya I., Imamoglu M., Isik A.U., Ozcan F. Carotid body tumors. Turk. J. Thorac. Cardiovasc. Surg. 1998; 6: 323-329.

6. Шубин А.А. Ангиохирургические аспекты лечения хемодектом шеи: Дисс. ... д-ра мед. наук. М., 2003. 314 c.

Shubin A.A. Angiosurgical aspects of treatment chemodectomas neck: Diss. ... Doct. of Med. Sci. Moscow, 2003. 314 p. (In Russian)

7. Sajid M.S., Hamilton G., Baker D.M.; Joint Vascular Research Group. A Multicenter review of carotid body tumour management. Eur. J. Vasc. Endovasc. Surg. 2007; 34 (2): 127-130.DOI:10.1016/j.ejvs.2007.01.015.

8. Welander J., Andreasson A., Brauckhoff M., Backdahl M., Larsson C., Gimm O., Söderkvist P. Frequent EPAS1/
HIF2 $\alpha$ exons 9 and 12 mutations in non-familial pheochromocytoma. Endocr. Relat. Cancer. 2014; 21 (3): 495-504. DOI: 10.1530/ERC-13-0384.

9. Von Haller. Cited by Gratiot J.H. Carotid tumors: A collective review. Abstr. Surg. 1943; 7: 117-186.

10. Glenner G.G., Grimley P. M. Tumors of the extraadrenal paraganglion system, including chemoreceptors // Atlas of Tumor Pathology. Washington, DC: U.S. Armed Forces Institute of Pathology, 1974: 13-33.

11. Zak F.G., Lawson W. Anatomy and topography in the paraganglionic chemoreceptor system. 1st ed. New York: Springer-Verlag, 1982: 15-49.

12. Persky M.S., Setton A., Niimi Y., Hartman J., Frank D., Berenstein A. Combined endovascular and surgical treatment of head and neck paragangliomas. A team approach. Head Neck. 2002; 24: 423-431. DOI:10.1002/hed.10068.

13. Lee J.H., Barich F., Karnell L.H., Robinson R.A., Zhen W.K., Gantz B.J., Hoffman H.T.; American College of Surgeons Commission on Cancer; American Cancer Society. National Cancer Data Base report on malignant paragangliomas of the head and neck. Cancer. 2002; 94 (3): 730-737. DOI 10.1002/cncr.10252.

14. Del Guercio L., Narese D., Ferrara D., Butrico L., Padricelli A., Porcellini M. Carotid and vagal body paragangliomas. Transl. Med. UniSa. 2013; 6 (6): 11-15.

15. Szymańska A., Szymański M., Czekajska-Chehab E., Gołabek W., Szczerbo-Trojanowska M. Diagnosis and management of multiple paragangliomas of the head and neck. Eur. Arch. Otorhinolaryngol. 2015; 272 (8): 1991-1999. DOI: 10.1007/s00405-014-3126-z.

16. Williams M.D., Tischler A.S. Update from the 4th Edition of the World Health Organization Classification of Head and Neck Tumours: Paragangliomas. Head Neck Pathol. 2017; 11 (1): 88-95. DOI: 10.1007/s12105-017-0786-1.

17. Davila V.J., Chang J.M., Stone W.M., Fowl R.J., Bower T.C., Hinni M.L., Money S.R. Current surgical management of carotid body tumors. J. Vasc. Surg. 2016; 64 (6): 1703-1710. DOI: 10.1016/j.jvs.2016.05.076.

18. Langerman A., Athavale S.M., Rangarajan S.V., Sinard R.J., Netterville J.L. Natural history of cervical paragangliomas: outcomes of observation of 43 patients. Arch Otolaryngol. Head Neck Surg. 2012; 1383 (4): 341-345.

DOI: $10.1001 /$ archoto.2012.37.

19. Papaspyrou K., Mewes T., Rossmann H., Fottner C., Schneider-Raetzke B., Bartsch O., Schreckenberger M., Lackner K.J., Amedee R.G., Mann W.J. Head and neck paragangliomas: Report of 175 patients (1989-2010). Head Neck. 2012; 34 (5): 632-637. DOI:10.1002/hed.21790.

20. Mhatre A.N., Li Y., Gasperin A., Lalwani A.K. SDHB, SDHC, and SDHD mutation screen in sporadic and familial head and neck paragangliomas. Clin. Genet. 2004; 66: 461-466. DOI: 10.1111/j.1399-0004.2004.00328.x.

21. Burnichon N., Brière J.J., Libè R., Vescovo L., Rivière J., Tissier F., Jouanno E., Jeunemaitre X., Bénit P., Tzagoloff A., Rustin P., Bertherat J., Favier J., GimenezRoqueplo A.P. SDHA is a tumor suppressor gene causing paraganglioma. Hum. Mol. Genet. 2010; 19: 3011-3020. DOI: $10.1093 / \mathrm{hmg} / \mathrm{ddq} 206$.

22. Martucci V., Pacak K. Pheochromocytoma and paraganglioma: diagnosis, genetics, management, and treatment. Curr. Probl. Cancer. 2014; 38 (1): 7-41. DOI:10.1016/j.currproblcancer.2014.01.001. 
23. Thabet M.H., Kotob H. Cervical paragangliomas: diagnosis, management and complications. J. Laryngol. Otol. 2001; 115: 467-474.

24. Lee K.Y., Oh Y.W., Noh H.J., Lee Y.J., Yong H.S., Kang E.U., Kim K.A., Lee N.J. Extraadrenal paragangliomas of the body: imaging features. Am. J. Roentgenol. 2006; 187 (2): 492-504. DOI: 10.2214/AJR.05.0370.

25. Myssiorek D., Ferlito A., Silver C.E., Rodrigo J.P., Baysal B.E., Fagan J.J., Suarez C., Rinaldo A. Screening for familial paragangliomas. Oral. Oncol. 2008; 44: 532-537. DOI: 10.1016/j.oraloncology.2007.06.010.

26. Jansen J.C., van den Berg R., Kuiper A., van der Mey A.G., Zwinderman A.H., Cornelisse C.J. Estimation of growth rate in patients with head and neck paragangliomas influences the treatment proposal. Cancer. 2000; 88: 2811-2816.

27. Атанасян Л.А. Хемодектома. М.: Медицина, 1969. 62 с. Atanasyan L. A. Chemodectoma. M.: Meditsina, 1969. 62 p. (In Russian)

28. O'Neill S., O'Donnell M., Harkin D., Loughrey M., Lee B., Blair P. A 22-year northern Irish experience of carotid body tumours. Ulster Med. J. 2011; 80 (3): 133-140.

29. Кармазановский Г.Г., Никитаев Н.С., Дан В.Н., Яшина Н.И., Шубин А.А., Щеголев А.И. Спиральная КТ в диагностике опухолей и других объемных образований в развилке сонных артерий. Медицинская визуализация. 2002; 1: 39-46.

Karmazanovsky G.G., NikitaevN.S., Dan V.N., Yashina N.I., Shubin A.A., Shchegolev A.I. Spiral CT in Diagnosis of Tumors and Volume Neoplasms in Bifurcation of Carotid Arteries. Medical Visualization. 2002; 1: 39-46. (In Russian)

30. Назимок Е.В., Сафронов С.Н., Крутова Т.В. Дифференциальная ультразвуковая диагностика каротидных хемодектом и других опухолевых образований шеи. Здравоохранение Таджикистана. 2013; 3: 61-66.

Nazimok E.V., Safronov S.N., Krutova T.V. Differential diagnosis of carotid chemodectoma and other tumors of the neck by ultrasound. Zdravoohranenie Tadzhikistana. 2013; 3: 61-66. (In Russian)

31. Jianu D.C., Jianu S.N., Motoc A.G., Dan T.F., Poenaru M., Tăban S., Cretu O.M. An evaluation on multidisciplinary management of carotid body paragangliomas: a report of seven cases. Rom. J. Morphol. Embryol. 2016; 57 (2): 853-859.

32. Berk M. Chemodektoma of the glomus intervagale: case report and review. Clin. Radial. 1961; 12: 219-226.

33. Аракелян В.С., Газимагомедов З.И., Абдулгасанов Р.А. Диагностика и результаты хирургического лечения каротидных хемодектом. Креативная кардиология. 2015; 2: 72-80. DOI: 10.15275/kreatkard.2015.02.07. Arakelyan V.S., Gazimagomedov Z.I., Abdulgasanov R.A. The results of diagnosis and surgical treatment of carotid chemodectomas. Kreativnaya kardiologiya. 2015; 2: 72-80. (InRussian). DOI: 10.15275/kreatkard.2015.02.07.

34. Белоцерковский И.В., Акинфеев В.В., Жуковец А.Г., Тризна Н.М. Хирургическое лечение параганглиом шеи. Опухоли головы и шеи. 2011; 3: 26-31.

Belotserkovsky I.V., Akinfeev V.V., Zhukovec A.G, Trizna N.M. Surgical treatment of the paragangliomas of the neck. Opukholi golovy i shei. 2011; 3: 26-31. (In Russian)

35. Газимагомедов 3.И. Параганглиомы шеи. Креативная кардиология. 2015; 1: 56-65.

DOI: 10.15275/kreatkard.2015.01.06.
Gazimagomedov Z.I. Paragangliomas of the neck. Kreativnaya kardiologiya. 2015; 1: 56-65. (In Russian) DOI: 10.15275/kreatkard.2015.01.06.

36. Lim J.Y., Kim J., Kim S.H., Lee S., Lim Y.C., Kim J.W., Choi E.C. Surgical treatment of carotid body paragangliomas: outcomes and complications according to the Shamblin classification. Clin. Exp. Otorhinolaryngol. 2010; 3: 91-95. DOI: 10.3342/ceo.2010.3.2.91.

37. Olsen W.L., Dillon W.P., Kelly W.M., Norman D., BrantZawadzki M., Newton T.H. MR imaging of paragangliomas. Am. J. Roentgenol. 1987; 148: 201-204. DOI:10.2214/ajr.148.1.201.

38. Wieneke J.A., Smith A. Paraganglioma: carotid body tumor. Head Neck Pathol. 2009; 3 (4): 303-306. DOI: 10.1007/s12105-009-0130-5.

39. Gravel G., Niccoli P., Rohmer V., Moulin G., BorsonChazot F., Rousset P., Pasco-Papon A., Marcus C., Dubrulle F., Gouya H., Bidault F., Dupas B., Gabrillargues J., Caumont-Prim A., Hernigou A., Gimenez-Roqueplo A., Halimi $P$. The value of a rapid contrast-enhanced angioMRI protocol in the detection of head and neck paragangliomas in SDHx mutations carriers: a retrospective study on behalf of the PGL.EVA investigators. Eur. Radiol. 2016; 26 (6): 1696-1704.

DOI 10.1007/s00330-015-4024-5.

40. van den Berg R., Verbist B.M., Mertens B.J., van der Mey A.G., van Buchem M.A. Head and neck paragangliomas: improved tumor detection using contrast-enhanced 3D time-of-flight MR angiography as compared with fatsuppressed MR imaging techniques. Am. J. Neuroradiol. 2004; 25 (5): 863-870.

41. Capatina C., Ntali G., Karavitaki N., Grossman A.B. The management of head-and neck paragangliomas. Endocr. Relat. Cancer. 2013; 20: 291-305. DOI: 10.1530/ERC-13-0223.

42. Hamersley E.R., Barrows A., Perez A., Schroeder A., Castle J.T. Malignant Vagal Paraganglioma. Head Neck. Pathol. 2016; 10 (2): 201-205. DOI: $10.1007 / \mathrm{s} 12105-015-0621-5$.

43. Tong Y. Role of duplex ultrasound in the diagnosis and assessment of carotid body tumour: A literature review. Intractable Rare Dis. Res. 2012; 1 (3): 129-133. DOI: 10.5582/irdr.v1.3.129.

44. White G., Forbes M., Busch K., Harris J.P., Makeham V. Carotid body tumour or not. Ultrasound diagnostic features. Vascular 2007 Conference Handbook \& Book of Abstracts. Melbourne, Australia, 2007. 72.

45. Offergeld C., Brase C., Yaremchuk S., Mader I., Rischke H.C., Gläsker S., Schmid K.W., Wiech T., Preuss S.F., Suárez C., Kopeć T., Patocs A., Wohllk N., Malekpour M., Boedeker C.C., Neumann H.P. Head and neck paragangliomas: clinical and molecular genetic classification. Clinics (Sao Paulo). 2012; 67: 19-28.

46. Dickens W.J., Million R.R., Cassisi N.J., Singleton G.T. Chemodectomas arising in temporal bone structures. Laryngoscope. 1982; 92 (2): 188-191.

47. GulyaA.J. The glomustumor and its biology. Laryngoscope. 1993; 103 (11, Pt 2, Suppl. 60): 7-15.

48. Brown J.S. Glomus jugulare tumors. Methods and difficulties of diagnosis and surgical treatment. Laryngoscope. 1967; 77 (1): 26-67. DOI: 10.1288/00005537-196701000-00004.

49. Mohan S., Hoeffner E., Bigelow D.C., Loevner L.A. Applications of magnetic resonance imaging in adult 
temporal bone disorders. Magn. Reson. Imaging Clin. N. Am. 2012; 20 (3): 545-572.

DOI: $10.1016 /$ j.mric.2012.06.001.

50. Subedi N., Prestwich R., Chowdhury F., Patel C., Scarsbrook A. Neuroendocrine tumours of the head and neck: anatomical, functional and molecular imaging and contemporary management. Cancer Imaging. 2013; 13 (3): 407-422. DOI:10.1102/1470-7330.2013.0034.

51. Devuyst L., Defreyne L., Praet M., Geukens S., Dhooge I. Treatment of glomus tympanicum tumors by preoperative embolization and total surgical resection. Am. J. Otolaryngol. 2016; 37 (6): 544-551.

DOI: 10.1016/j.amjoto.2016.08.011.

52. Neves F., Huwart L., Jourdan G., Reizine D., Herman P., Vicaut E., Guichard J.P. Head and neck paragangliomas: value of contrast-enhanced 3D MR angiography. Am. J. Neuroradiol. 2008; 29: 883-889.

DOI: 10.3174/ajnr.A0948.

53. Luna-Ortiz K., Rason-Ortiz M., Villavicencio-Valencia V., Herrera-Gomez A. Does Shamblin's classification predict postoperative morbidity in carotid body tumors? A proposal to modify Shamblin's classification? Eur. Arch. Otorhinolaryngol. 2006; 2: 171-175.

DOI: 10.1007/s00405-005-0968-4.

54. Reigner. Cited by Lahey F.H., Warren K.W. A long-term appraisal of carotid tumors with remarks on their removal. Surg. Gynecol. Obstet. 1951; 92: 481-491.

55. Maydl. Cited by Byme J.J. Carotid body and allied tumors. Am. J. Surg. 1958; 95: 371-384.

56. Muhm M., Polterauer P., Gstottner W., Temmel A., Richling B., Undt G., Niederle B., Staudacher M., Ehringer $\mathrm{H}$. Diagnostic and therapeutic approaches to carotid body tumors: review of 24 patients. Arch. Surg. 1997; 132 (3): 279-284.

57. Gordon-Taylor G. On carotid tumours. Br. J. Surg. 1940; 28 (110): 163-172.

58. Shamblin W.R., Remine W.H., Sheps S.G., Harrison E.G. Carotid body tumor (chemodectoma). Clinicophatologic analysis of 90 cases. Am. J. Surg. 1971; 122 (6): 732-739.

59. Ma Y., Huang D., Liu L., Xiang M., Oghagbon E.K., Zhai S. Surgical treatment of carotid body tumour: a report of 39 cases and a new classification of carotid body tumour: our experience. Clin. Otolaryngol. 2014; 39 (4): 254-257. DOI: 10.1111/coa.12267.
60. Дан В.Н., Матякин Е.Г., Коков Л.С., Кармазановский Г.Г. Предварительная эмболизация при хирургическом лечении хемодектом шеи. Ангиология и сосудистая хирургия. 2000; 6 (2): 109-115.

Dan V.N., Matjakin E.G., Kokov L.S., Karmazanovsky G.G. Preliminary embolization during surgical treatment with chemodectoma of the neck. Angiologiya $i$ sosudistaya khirurgiya. 2000; 6 (2): 109-115. (In Russian)

61. Jackson R.S., Myhill J.A., Padhya T.A., McCaffrey J.C., McCaffrey T.V., Mhaskar R.S. The effects of preoperative embolization on carotid body paragangliomas surgery: a systematic review and meta-analysis. Otolaryngol. Head. Neck. Surg. 2015; 153 (6): 943-50. DOI: $10.1177 / 0194599815605323$.

62. Dixon J.L., Atkins M.D., Bohannon W.T., Buckley C.J., Lairmore T.C. Surgical management of carotid body tumors: a 15-year single institution experience employing an interdisciplinary approach. Proc (Bayl. Univ. Med. Cent). 2016; 29 (1): 16-20.

63. Power A.H., Bower T.C., Kasperbauer J., Link M.J., Oderich G., Cloft H., Young WF.Jr., Gloviczki P. Impact of preoperative embolization on outcomes of carotid body tumor resections. J. Vasc. Surg. 2012; 56: 979-989. DOI: $10.1016 /$ j.jvs.2012.03.037.

64. Bercin S., Muderris T., Sevil E., Gul F., Kilicarslan A., Kiris M. Efficiency of preoperative embolization of carotid body tumor. Auris Nasus Larynx. 2015; 42 (3): 226-230. DOI: 10.1016/j.anl.2014.10.013.

65. Bianchi L.C., Marchetti M., Brait L., Bergantin A., Milanesi I., Broggi G., Fariselli L. Paragangliomas of head and neck: a treatment option with Cyberknife radiosurgery. Neurol. Sci. 2009; 30: $479-485$. DOI: 10.1007/s10072-009-0138-3.

66. Galland-Girodet S., Maire J.P., De-Mones E., Benech J., Bouhoreira K., Protat B., Demeaux H., Darrouzet V., Huchet $A$. The role of radiation therapy in the management of head and neck paragangliomas: Impact of quality of life versus treatment response. Radiother. Oncol. 2014; 111 (3): 463-467. DOI: 10.1016/j.radonc.2014.06.002.

67. Guss Z.D., Batra S., Limb C.J., Li G., Sughrue M.E., Redmond K., Rigamonti D., Parsa A.T., Chang S., Kleinberg L., Lim M. Radiosurgery of glomus jugulare tumors: a meta-analysis. Int. J. Radiation. Oncol. Biol. Phys. 2011; 81: 497-502. DOI: 10.1016/j.jjrobp.2011.05.006. 